\title{
Provider Attitudes and Practice Patterns for Direct-Acting Antiviral Therapy for Patients With Hepatocellular Carcinoma
}

\author{
Nicole E. Rich, ${ }^{*}$ Ju Dong Yang, ${ }^{\ddagger}$ Ponni V. Perumalswami, ${ }^{\S}$ Naim Alkhouri, \\ Whitney Jackson, ${ }^{, ~ N e e h a r ~ D . ~ P a r i k h, ~}{ }^{\#}$ Neil Mehta, ${ }^{* \star}$ Reena Salgia, ${ }^{\text {} \neq}$ \\ Andres Duarte-Rojo, ${ }^{\S \S}$ Laura Kulik, \|\|$"$ Mina Rakoski, " \\ Omobonike Oloruntoba, ${ }^{\star \star \star}$ George N. loannou, ${ }^{\neq \neq \ddagger}$ Maarouf A. Hoteit, $\$ \S \S$

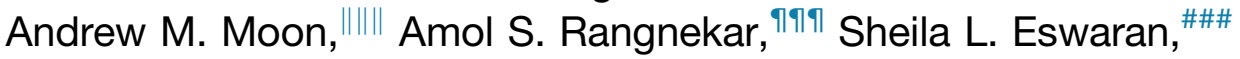 \\ Elizabeth Zheng, ${ }^{\star \star \star \star}$ Janice H. Jou, ${ }^{\neq \neq \neq \neq}$James Hanje, ${ }^{\S \S \S}$ Anjana Pillai, $\||||| \mid$ \\ Ruben Hernaez, " \\ Hrishikesh Samant,

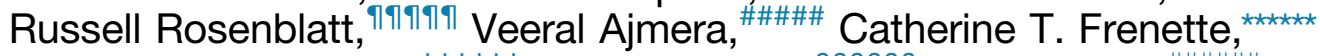 \\ Sanjaya K. Satapathy, ${ }^{\ddagger \neq \neq \neq \neq \ddagger}$ Parvez Mantry, ${ }^{\S \S \S \S \S \S}$ Prasun Jalal, $\||\|||||| \mid$ \\ Binu V. John, ,

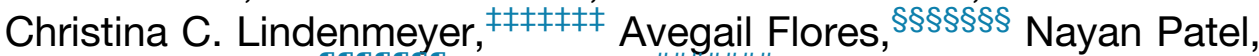

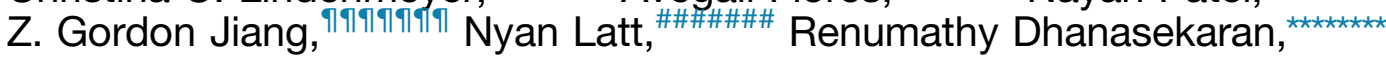 \\ Mobolaji Odewole, ${ }^{*}$ Sofia Kagan, ${ }^{*}$ Jorge A. Marrero, ${ }^{*}$ and Amit G. Singal ${ }^{\star}$
}

${ }^{*}$ Division of Digestive and Liver Disease, UT Southwestern Medical Center, Dallas, Texas; ${ }^{\ddagger}$ Division of Digestive and Liver Diseases, Comprehensive Transplant Center and Samuel Oschin Comprehensive Cancer Institute, Cedars Sinai Medical Center, Los Angeles, California; \$Division of Liver Diseases, Icahn School of Medicine at Mount Sinai, New York, New York;

"Texas Liver Institute, University of Texas Health San Antonio, San Antonio, Texas; "Division of Gastroenterology and Hepatology, University of Colorado Denver School of Medicine, Denver, Cólorado; "Division of Gastroenterology and Hepatology, University of Michigan, Ann Arbor, Michigan; ${ }^{* *}$ Division of Gastroenterology, University of California San Francisco, San Francisco, California; ${ }^{\ddagger \ddagger}$ Division of Gastroenterology and Hepatology, Henry Ford Hospital, Detroit, Michigan; ${ }^{\S}$ T.E. Starzl Transplantation Institute and Center for Liver Disease, University of Pittsburgh Medical Center, Pittsburgh, Pennsylvania;

"II Division of Hepatology, Northwestern University, Chicago, Illinois; ${ }^{19}$ Transplantation Institute and Division of Gastroenterology, Loma Linda University Health, Loma Linda, California; "\#ivision of Gastroenterology and Hepatology, University of Wisconsin School of Medicine, Madison, Wisconsin; ${ }^{* * *}$ Division of Gastroenterology and Hepatology, Duke University Health Center, Durham, North Carolina; ${ }^{\ddagger \neq}$ Division of Gastroenterology and Research and Development, Veterans Affairs Puget Sound Healthcare System and University of Washington, Seattle, Washington; ${ }^{\S \S D i v i s i o n ~ o f ~ G a s t r o e n t e r o l o g y ~}$ and Hepatology, University of Pennsylvania, Philadelphia, Pennsylvania; "IIIIDivision of Gastroenterology and Hepatology, University of North Carolina School of Medicine, Chapel Hill, North Carolina; "17ा Division of Gastroenterology, Georgetown

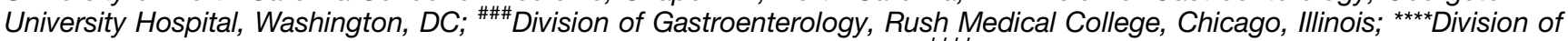
Digestive and Liver Diseases, Columbia University, New York, New York; ${ }^{\ddagger \ddagger \ddagger}$ Division of Gastroenterology and Hepatology, Oregon Health and Science University, Portland, Oregon; §§§§Division of Gastroenterology, Hepatology and Nutrition, The Ohio State University Wexner Medical Center, Columbus, Ohio; "IIIII Division of Gastroenterology, Hepatology and Nutrition, University of Chicago, Chicago, Illinois; ${ }^{17 / 19 / S e c t i o n ~ o f ~ G a s t r o e n t e r o l o g y ~ a n d ~ H e p a t o l o g y, ~ B a y l o r ~ C o l l e g e ~ o f ~ M e d i c i n e ~ a n d ~}$ Michael E. Debakey Veterans Affairs Medical Center, Houston, Texas; "\#\#\# Division of Gastroenterology and Hepatology,

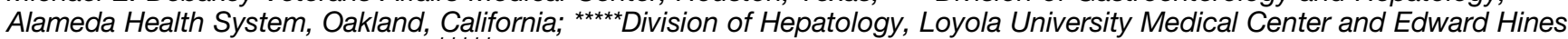
Veterans Affairs, Chicago, Illinois; ${ }^{\ddagger \neq \neq \neq \ddagger D i v i s i o n ~ o f ~ G a s t r o e n t e r o l o g y ~ a n d ~ H e p a t o l o g y, ~ L o u i s i a n a ~ S t a t e ~ U n i v e r s i t y ~ H e a l t h ~}$

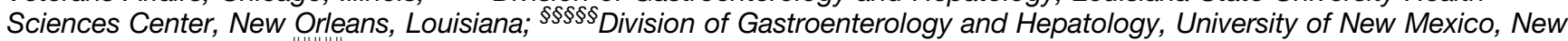
Mexico, Albuquerque; $\|||||||$ Division of Gastroenterology, Hepatology and Nutrition, University of Utah, Salt Lake City, Utah; १191979 Division of Gastroenterology and Hepatology, Weill Cornell Medicine - New York-Presbyterian Hospital, New York, New

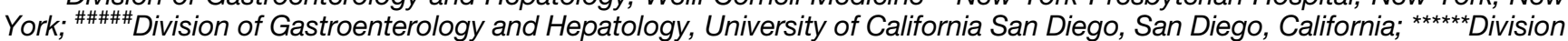
of Organ Transplantation, Scripps Green Hospital, San Diego, California; ${ }^{\ddagger \ddagger \ddagger \neq \ddagger}$ Division of Transplant Surgery, University of

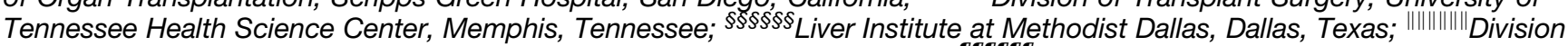
of Abdominal Transplantation, Baylor College of Medicine, Houston, Texas; 19191919 Division of Gastroenterology and Hepatology, McGuire Veterans Affairs Medical Center, Richmond, Virginia; "\#\#\#\#\# Organ Transplant Department, Swedish Medical Center, Seattle, Washington; ${ }^{* * * * * *}$ Division of Gastroenterology and Hepatology, Mayo Clinic, Rochester, Minnesota;

Abbreviations used in this paper: APP, advanced practice provider; DAA, direct-acting antiviral; F3, stage 3 fibrosis; HCC, hepatocellular carcinoma; HCV, hepatitis C virus; SVR, sustained virologic response. 


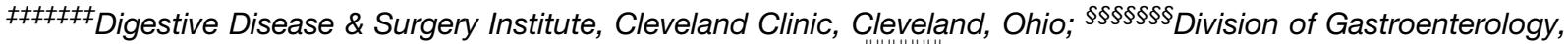
Washington University School of Medicine, St. Louis, Missouri; |||||||||||||Banner Transplant Institute, Banner - University Medical Center Phoenix, Phoenix, Arizona; ${ }^{1791791791 D i v i s i o n ~ o f ~ G a s t r o e n t e r o l o g y ~ a n d ~ H e p a t o l o g y, ~ B e t h ~ I s r a e l ~ D e a c o n e s s ~ M e d i c a l ~ C e n t e r, ~}$ Boston, Massachusetts; "\#\#\#\#\#\# Oschner Multi-Organ Transplant Institute, Oschner Health System, New Orleans, Louisiana; and

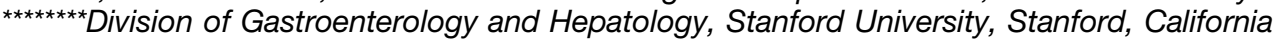

BACKGROUND \& AIMS: Direct-acting antivirals (DAAs) are effective against hepatitis C virus and sustained virologic response is associated with reduced incidence of hepatocellular carcinoma (HCC). However, there is controversy over the use of DAAs in patients with active or treated HCC and uncertainty about optimal management of these patients. We aimed to characterize attitudes and practice patterns of hepatology practitioners in the United States regarding the use of DAAs in patients with HCC.

METHODS:

We conducted a survey of hepatology providers at 47 tertiary care centers in 25 states. Surveys were sent to 476 providers and we received 279 responses $(58.6 \%)$.

RESULTS:

Provider beliefs about risk of HCC recurrence after DAA therapy varied: $\mathbf{4 8 \%}$ responded that DAAs reduce risk, $36 \%$ responded that DAAs do not change risk, and $16 \%$ responded that DAAs increase risk of HCC recurrence. However, most providers believed DAAs to be beneficial to and reduce mortality of patients with complete response to HCC treatment. Accordingly, nearly all providers $(94.9 \%)$ reported recommending DAA therapy to patients with earlystage HCC who received curative treatment. However, fewer providers recommended DAA therapy for patients with intermediate (72.9\%) or advanced (57.5\%) HCC undergoing palliative therapies. Timing of DAA initiation varied among providers based on HCC treatment modality: $49.1 \%$ of providers reported they would initiate DAA therapy within 3 months of surgical resection whereas $45.9 \%$ and 5.0\% would delay DAA initiation for 3-12 months and $>1$ year post-surgery, respectively. For patients undergoing transarterial chemoembolization (TACE), 42.0\% of providers would provide DAAs within 3 months of the procedure, $46.7 \%$ would delay DAAs until 3-12 months afterward, and $11.3 \%$ would delay DAAs more than 1 year after TACE.

CONCLUSIONS: $\quad$ Based on a survey sent to hepatology providers, there is variation in provider attitudes and practice patterns regarding use and timing of DAAs for patients with HCC. Further studies are needed to characterize the risks and benefits of DAA therapy in this patient population.

Keywords: Liver Cancer; HCV; TACE; Drug.

$\mathrm{H}$ epatocellular carcinoma (HCC) is the fastest rising cause of cancer-related death in the United States, with most cases attributed to chronic hepatitis $\mathrm{C}$ virus (HCV) infection. ${ }^{1-3}$ Highly effective direct-acting antivirals (DAAs) have revolutionized HCV treatment, resulting in high rates of sustained virologic response (SVR). HCV eradication in patients with cirrhosis with DAAs is cost-effective ${ }^{4}$ and has several benefits including reduced risk of hepatic decompensation, $^{5}$ improvement in all-cause mortality, ${ }^{6,7}$ and decreased incident $\mathrm{HCC}^{7-9}$

However, the effect of DAAs on risk of tumor recurrence in patients with a history of treated HCC remains controversial after an observational study from Spain found a higher-than-expected proportion of patients with HCC recurrence after DAA treatment. ${ }^{10}$ Subsequent studies have produced conflicting data, including a large multicenter study showing no significant difference in early HCC recurrence, overall recurrence, or tumor aggressiveness between DAAtreated and untreated patients. ${ }^{11}$ Furthermore, there are unknown benefits of DAA treatment in patients with active HCC, particularly in light of reports showing reduced rates of SVR. ${ }^{12,13}$

While awaiting prospective data, there remains uncertainty about the optimal management of patients with HCV and HCC, which may lead to confusion among providers. To our knowledge, provider attitudes and practice patterns in this patient population have not been assessed. Therefore, we conducted a nationwide survey study to evaluate attitudes and practice patterns of US hepatology practitioners regarding the use and timing of DAA treatment in patients with HCC.

\section{Methods}

\section{Participants}

We conducted a survey among hepatology providers at 47 tertiary care centers, safety-net hospitals, and Veterans Affairs hospitals from 25 states in the United States. Participating sites were a convenience sample of academic centers, representing each US region. A single 
provider at each site, whose email was obtained from their institutional Web site, was asked to distribute the survey to all hepatology providers at his or her institution. Eligible providers included physicians (MD/DO) and advanced practice providers (APPs) involved in clinical care of patients with HCV. We excluded providers who primarily treat patients $<18$ years of age, nonhepatology providers, and those with incomplete surveys. The study was approved by the institutional review board at the University of Texas Southwestern Medical Center.

\section{Survey Information}

We distributed an anonymous web-based survey to eligible hepatology providers between February 1, 2019 and February 28, 2019. We sent a single email reminder to those who had not completed the survey after 1-2 weeks. The survey had 38 questions and took an average of 10 minutes to complete.

The content of the survey was based on a conceptual model, adapted from a previously proposed model of physician behavior (Figure 1). ${ }^{14}$ Survey questions were organized into 5 sections:

1. Provider experience (4 questions): assessed provider experience with HCV and HCC treatment.

2. Provider practice patterns (7 questions): assessed provider practice patterns with regard to $\mathrm{HCV}$ treatment, HCC treatment, and HCC surveillance.

3. Clinical vignettes (10 questions): included 4 clinical vignettes to assess provider practices for patients with different stages of HCC undergoing curative and palliative HCC treatments.

4. Provider attitudes and beliefs (9 questions): assessed provider attitudes and beliefs about potential benefits and risks of DAA therapy in patients with HCC.

5. Provider demographics (8 questions): recorded provider demographics including age, sex, race/ ethnicity, number of years in clinical practice,

\section{What You Need to Know}

\section{Background}

The use and timing of direct acting antivirals (DAAs) for patients with a history of treated hepatocellular carcinoma (HCC) is controversial due to concerns about risk of HCC recurrence.

\section{Findings}

In a survey of 279 hepatology practitioners, we found variation in perceived risk of HCC recurrence after DAA therapy. Most providers believe DAAs reduce mortality in patients with a history of complete response to HCC therapy. Recommendations for DAA therapy in HCC patients varied by tumor stage, with fewer providers recommending DAAs for patients with a history of intermediate or advanced HCC than early HCC. Timing of DAA therapy also varied among providers, with some starting DAA around time of HCC treatment and others delaying DAA for months to confirm complete response to HCC treatment.

\section{Implications for patient care}

Future studies characterizing the risks and benefits of DAAs for patients with a history of HCC might help standardize clinical practice for these patients.

provider type (physician vs APP), presence of an institutional multidisciplinary tumor board, and number of patients treated annually with HCV and HCC.

Questions were adapted from validated surveys when available. ${ }^{15}$ The survey was pretested among 5 MD providers, at which time saturation of feedback was believed to have been achieved. Each provider participated in a cognitive interview after survey completion, and the survey was iteratively revised based on feedback before distribution to study participants. The complete survey can be found in the Supplementary Material.

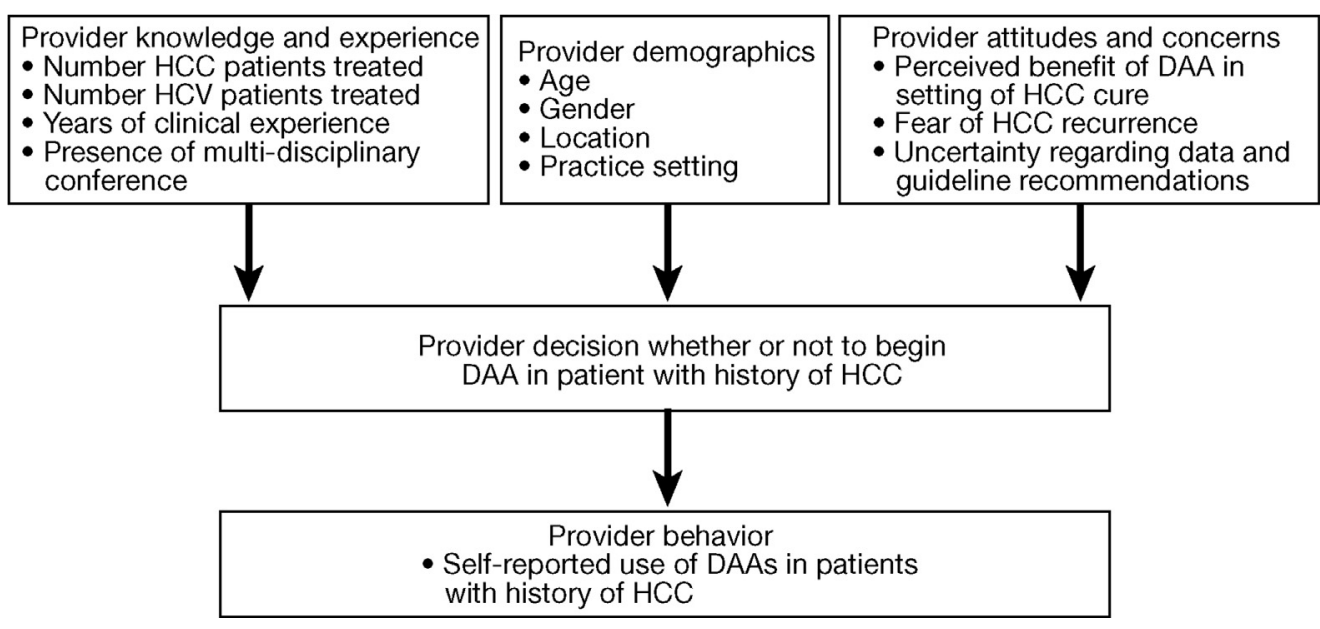

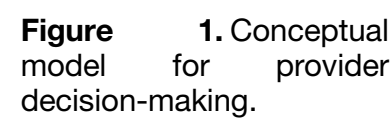




\section{Statistical Analysis}

Survey responses were summarized using descriptive statistics. The primary outcome of interest was provider-reported practice patterns for DAA recommendations in patients with HCC, based on provider responses to 4 clinical vignettes. Secondary outcomes included provider attitudes regarding the use of DAAs in patients with HCC. Fisher exact and chi-square tests were performed for categorical variables to identify factors associated with DAA recommendation patterns. Independent variables included provider demographics and perceived DAA benefit in patients with HCC. Tests were 2-sided and performed at the 5\% significance level. Statistical analysis was performed using Stata version 14.0 (College Station, TX).

\section{Results}

\section{Provider and Institutional Characteristics}

Of 331 surveys returned, 52 were excluded because of being incomplete, leaving a total of 279 responses. This represented a $58.6 \%$ (279 of 476 ) provider-level and $92.2 \%$ (47 of 51) institution-level response rate. Characteristics of respondents are summarized in Table 1. Most respondents were MD or DO providers, over $50 \%$ were female, and the sample was racially and ethnically diverse. Most providers identified their primary practice location as a tertiary referral center with a transplant program, approximately threefourths had been in practice for $>5$ years, and nearly two-thirds spent $>75 \%$ of time on clinical care. More than $75 \%$ of providers had treated more than 50 total patients with HCV with DAAs, and $90 \%$ reported being directly involved in HCC management.

\section{Provider Attitudes Regarding Use of Direct- Acting Antivirals in Patients With Hepatocellular Carcinoma}

Provider-reported attitudes regarding the use of DAAs in the setting of HCC are reported in Table 2. Nearly $80 \%$ of providers believed DAAs reduce risk of incident de novo HCC in patients with cirrhosis. However, there was wider variation in perceived risk of HCC recurrence after DAA therapy in patients with complete response to HCC treatment, with $48 \%$ believing DAAs reduce risk of recurrence, 36\% believing DAAs do not change HCC recurrence risk, and $16 \%$ believing DAAs increase recurrence risk. Despite this, most still believed DAAs reduce mortality, are cost-effective, and have overall benefit in patients with complete response to HCC treatment. In fact, nearly all providers were likely to recommend DAAs in patients with a history of HCC, although $50 \%$
Table 1. Provider and Institution Characteristics $(n=279)$

\begin{tabular}{|c|c|}
\hline Characteristics & $\mathrm{N}(\%)$ \\
\hline \multicolumn{2}{|l|}{ Provider } \\
\hline Sex (\% male) & $133(48.4)$ \\
\hline \multicolumn{2}{|l|}{ Race/ethnicity } \\
\hline Non-Hispanic white & $146(53.3)$ \\
\hline Black & $4(1.5)$ \\
\hline Hispanic & $11(4.0)$ \\
\hline Asian/Pacific Islander & $81(29.6)$ \\
\hline Other & $32(11.7)$ \\
\hline \multicolumn{2}{|l|}{ Type of practitioner } \\
\hline Physician (MD/DO) & $195(70.1)$ \\
\hline APP & $83(29.9)$ \\
\hline \multicolumn{2}{|l|}{ Years in practice } \\
\hline$<5$ & $71(25.5)$ \\
\hline $5-9$ & $84(30.2)$ \\
\hline $10-20$ & $75(27.0)$ \\
\hline$>20$ & $48(17.3)$ \\
\hline \multicolumn{2}{|l|}{ Time spent on clinical care, $\%$} \\
\hline$<50$ & $46(16.6)$ \\
\hline $50-75$ & $57(20.5)$ \\
\hline$>75$ & $175(62.9)$ \\
\hline \multicolumn{2}{|l|}{$\begin{array}{l}\text { Approximate total number patients with HCV } \\
\text { treated with DAAs }\end{array}$} \\
\hline$<50$ & $57(20.5)$ \\
\hline $50-100$ & $57(20.5)$ \\
\hline$>100$ & $164(59.0)$ \\
\hline $\begin{array}{l}\text { Personally involved in HCC management (\% yes) } \\
\text { Institution }\end{array}$ & $253(91.3)$ \\
\hline \multicolumn{2}{|l|}{ Region } \\
\hline Northeast & $48(17.2)$ \\
\hline Midwest & $72(25.8)$ \\
\hline South & $76(27.2)$ \\
\hline West & $83(29.8)$ \\
\hline \multicolumn{2}{|l|}{ Institution type } \\
\hline Tertiary referral center with transplant program & $245(88.4)$ \\
\hline Tertiary referral center without transplant program & $8(2.9)$ \\
\hline Veterans Affairs hospital & $9(3.3)$ \\
\hline Community-based hospital & $7(2.5)$ \\
\hline Safety-net hospital & $8(2.9)$ \\
\hline \multicolumn{2}{|l|}{$\begin{array}{l}\text { Approximate number of patients with } \mathrm{HCC} \text { treated at } \\
\text { institution annually }\end{array}$} \\
\hline $0-50$ & $19(6.9)$ \\
\hline $51-100$ & $57(20.8)$ \\
\hline $101-150$ & $58(21.2)$ \\
\hline$>150$ & $140(51.1)$ \\
\hline $\begin{array}{l}\text { Presence of multidisciplinary HCC clinic and/or } \\
\text { conference (\% yes) }\end{array}$ & $274(98.6)$ \\
\hline
\end{tabular}

APP, advanced practice provider; DAA, direct-acting antiviral; DO, Doctor of Osteopathic Medicine; HCC, hepatocellular carcinoma; HCV, hepatitis $\mathrm{C}$ virus; $\mathrm{MD}$, Medical Doctor.

delayed initiation of DAA therapy by 4-6 months following HCC complete response. Only $5 \%$ of providers believed HCC recurrence after DAA therapy could pose legal liability, compared with more than one-third believing this was possible in untreated patients who experience hepatic decompensation. However, $60 \%$ of providers reported counseling their patients regarding the risk of HCC recurrence before DAA therapy. Overall, $85 \%$ of providers believed this is an area of continued controversy in need of more data and guidance. 
Table 2. Provider Attitudes Regarding Use of DAAs in Patients With HCC $(n=279)$

\begin{tabular}{|c|c|}
\hline Provider attitude & $\mathrm{N}(\%)$ \\
\hline \multicolumn{2}{|l|}{$\begin{array}{l}\text { Impact of DAA on incident HCC risk in patients } \\
\text { with cirrhosis }\end{array}$} \\
\hline Significant reduction in risk & $167(60.1)$ \\
\hline Small reduction in risk & $53(19.1)$ \\
\hline No change in risk & $41(14.7)$ \\
\hline Small increase in risk & $15(5.4)$ \\
\hline Significant increase in risk & $2(0.7)$ \\
\hline \multicolumn{2}{|l|}{$\begin{array}{l}\text { Impact of DAA on risk of HCC recurrence in patients } \\
\text { with CR to HCC treatment }\end{array}$} \\
\hline Significant reduction in risk & $46(16.7)$ \\
\hline Small reduction in risk & $85(30.8)$ \\
\hline No change in risk & $99(36.0)$ \\
\hline No change in risk but shortens time-to-recurrence & $15(5.4)$ \\
\hline Small increase in risk & $30(10.9)$ \\
\hline Significant increase in risk & $0(0.0)$ \\
\hline \multicolumn{2}{|l|}{$\begin{array}{l}\text { Impact of DAA on mortality in patients with } \\
\text { CR to HCC treatment }\end{array}$} \\
\hline Significant reduction in mortality & $104(37.8)$ \\
\hline Small reduction in mortality & $110(40.0)$ \\
\hline No change in mortality & $56(20.4)$ \\
\hline Small increase in mortality & $4(1.4)$ \\
\hline Significant increase in mortality & $1(0.4)$ \\
\hline \multicolumn{2}{|l|}{$\begin{array}{l}\text { Impact of active HCC on likelihood of achieving } \\
\text { SVR with DAA treatment }\end{array}$} \\
\hline Significant reduction in SVR rates & $57(20.4)$ \\
\hline Small reduction in SVR rates & $114(40.9)$ \\
\hline No change in SVR rates & $104(37.3)$ \\
\hline Small increase in SVR rates & $4(1.4)$ \\
\hline Significant increase in SVR rates & $0(0.0)$ \\
\hline \multicolumn{2}{|l|}{$\begin{array}{l}\text { DAAs are cost-effective in patients with } \\
\text { CR to HCC treatment }\end{array}$} \\
\hline Strongly agree & $114(40.9)$ \\
\hline Agree & $151(54.1)$ \\
\hline Disagree & $13(4.7)$ \\
\hline Strongly disagree & $1(0.4)$ \\
\hline \multicolumn{2}{|l|}{$\begin{array}{l}\text { Overall, DAAs are beneficial in patients with } \\
\text { CR to HCC treatment }\end{array}$} \\
\hline Strongly agree & $133(47.7)$ \\
\hline Agree & $139(49.8)$ \\
\hline Disagree & $6(2.1)$ \\
\hline Strongly disagree & $1(0.4)$ \\
\hline \multicolumn{2}{|l|}{$\begin{array}{l}\text { Treating patients with HCC with DAAs may pose legal } \\
\text { liability if patients have HCC recurrence }\end{array}$} \\
\hline Strongly agree & $2(0.7)$ \\
\hline Agree & $15(5.4)$ \\
\hline Disagree & $161(57.7)$ \\
\hline Strongly disagree & $101(36.2)$ \\
\hline \multicolumn{2}{|c|}{$\begin{array}{l}\text { Not treating patients with } \mathrm{HCC} \text { with DAAs may pose legal } \\
\text { liability if patients develop hepatic decompensation }\end{array}$} \\
\hline Strongly agree & $27(9.7)$ \\
\hline Agree & $80(28.8)$ \\
\hline Disagree & $134(48.2)$ \\
\hline Strongly disagree & 37 (13.3) \\
\hline \multicolumn{2}{|l|}{$\begin{array}{l}\text { More data and guidance are needed on risk of HCC } \\
\text { recurrence after DAAs }\end{array}$} \\
\hline Strongly agree & $117(41.9)$ \\
\hline Agree & $122(43.7)$ \\
\hline Disagree & $35(12.5)$ \\
\hline Strongly disagree & $5(1.8)$ \\
\hline
\end{tabular}

Table 2. Continued

\begin{tabular}{lc}
\hline \multicolumn{1}{c}{ Provider attitude } & N (\%) \\
\hline How have recent studies on DAA and HCC risk & \\
changed your clinical practice? & $0(0.0)$ \\
No longer use DAAs in patients with HCC & $12(4.4)$ \\
Less likely to use DAAs in patients with HCC & $138(50.6)$ \\
Equally likely to use DAAs but I delay therapy 4-6 mo & \\
$\quad$ after CR & \\
Equally likely to use DAAs with no change in my practice & $123(45.0)$ \\
Does HCC treatment modality that led to CR impact your & \\
$\quad$ decision regarding timing of DAA? & $64(23.5)$ \\
Yes & $192(70.6)$ \\
No & $8(2.9)$ \\
Not applicable, I use DAA in patients with active HCC & $8(2.9)$ \\
Other & \\
How often do you counsel patients with HCC on risk of & \\
recurrence after DAA? & $119(43.4)$ \\
Always & $50(18.2)$ \\
Sometimes & $49(17.9)$ \\
Rarely & $56(20.4)$ \\
Never & $15(5.3)$ \\
Timing of DAA therapy in transplant candidate with HCC & \\
Routinely treat pretransplant on waitlist & $119(41.9)$ \\
Case-by-case basis & $122(43.0)$ \\
Routinely treat with DAA within 3 mo post-transplant & $298(9.9)$ \\
Routinely defer DAA >6 mo post-transplant &
\end{tabular}

$\mathrm{CR}$, complete response; DAA, direct-acting antiviral; HCC, hepatocellular carcinoma; SVR, sustained virologic response.

MD and DO providers were significantly more likely than APPs to believe that DAAs reduce risk of incident HCC (84.5\% vs $66.3 \% ; P=.002$ ); however, attitudes about HCC incidence or recurrence did not otherwise differ by provider sex, race/ethnicity, years in practice, provider experience with HCV or HCC treatment, type of institution, or region of the United States in which they practiced.

\section{Provider Attitudes Regarding Hepatocellular Carcinoma Surveillance After Sustained Virologic Response}

Surveillance practices in patients without a history of HCC are shown in Table 3. More than $90 \%$ of providers continue to follow post-SVR patients with cirrhosis in their clinics and perform HCC surveillance with imaging $\pm \alpha$-fetoprotein every 6 months indefinitely. Nearly $95 \%$ reported performing HCC surveillance in some patients with stage 3 fibrosis (F3), with $61 \%$ performing surveillance in all $\mathrm{F} 3$ patients.

\section{Provider Practices Regarding Use of Direct- Acting Antivirals in Patients With Hepatocellular Carcinoma}

Provider practice patterns for DAA use and DAA timing among patients undergoing HCC therapy are 
Table 3. HCC Surveillance Practice Patterns in Post-SVR Patients $(n=279)$

\begin{tabular}{lr}
\hline \multicolumn{1}{c}{ Provider practice } & N (\%) \\
\hline Length of time patients with cirrhosis with SVR & \\
post-DAAs are followed in hepatology clinic & $255(91.7)$ \\
Indefinitely & $9(3.2)$ \\
Approx. 1 y then discharge to PCP & $6(2.2)$ \\
Approx. 3-5 y then discharge to PCP & $9(2.9)$ \\
Not routinely followed; seen as needed & \\
Method of HCC surveillance in patients with & \\
cirrhosis after SVR & \\
Imaging (US, CT, or MR) \pm AFP every 6 mo indefinitely & $264(95.0)$ \\
Imaging (US, CT, or MR) \pm AFP every 12 mo indefinitely & $8(2.9)$ \\
Imaging (US, CT, or MR) \pm AFP every 6 mo, stop after & $2(0.7)$ \\
$\quad$ few years & $4(1.4)$ \\
Other & \\
Do you perform HCC surveillance in patients & \\
with F3 after SVR? & $169(61.0)$ \\
Yes & $91(32.9)$ \\
Sometimes & $17(6.1)$ \\
No &
\end{tabular}

AFP, $\alpha$-fetoprotein; CT, computed tomography; DAA, direct-acting antivirals; F3, stage 3 fibrosis; HCC, hepatocellular carcinoma; MR, magnetic resonance imaging; PCP, primary care physician; SVR, sustained virologic response; US, ultrasound.

illustrated in Figure $2 A$ and Figure $2 B$, respectively. Based on the clinical vignettes, the proportion of providers recommending DAA therapy and timing of such therapy varied by HCC stage and treatment type. Providers were significantly less likely to recommend DAAs in patients with intermediate- or advanced-stage HCC undergoing palliative treatments than early stage patients with HCC undergoing curative therapy. Although nearly 95\% would recommend DAAs in a patient with early stage HCC undergoing surgical resection, this was reported by only $73 \%$ and $57 \%$ of providers for intermediate-stage patients with HCC undergoing transarterial chemoembolization and advanced-stage patients with HCC undergoing systemic therapy, respectively. However, providers seemed willing to treat patients with more advanced HCC if they had a response to HCCdirected therapies, with nearly $40 \%$ willing to treat advanced-stage patients with HCC if they had an objective response to treatment. Nearly $70 \%$ of providers reported the modality leading to an HCC complete response would not impact their likelihood to recommend DAA therapy. Provider responses varied with regard to timing of DAA initiation based on HCC stage and treatment modality as demonstrated in Table 4. Overall, $15 \%-20 \%$ of providers reported they would initiate DAA therapy before HCC treatment; however, more than $50 \%$ delayed DAA initiation at least 3 months and 5\%-10\% delayed initiation more than 1 year. In patients with HCC listed for liver transplantation, only $5 \%$ of providers reported routinely treating patients with DAAs while on the waiting list, with most (53\%) treating HCV posttransplant or on a case-by-case basis (42\%).
Physicians (MDs and DOs) seemed less likely than APPs to treat patients with HCC undergoing palliative therapies; the difference did not reach statistical significance for patients undergoing transarterial chemoembolization $(55.7 \%$ vs $62.2 \% ; P=.32)$ but was significantly lower in patients undergoing systemic therapy (68.4\% vs $83.1 \% ; P=.01)$. Providers were also more likely to recommend DAAs in patients receiving systemic therapy if they reported fearing potential legal liability for untreated patients who experience hepatic decompensation $(65.4 \%$ vs $52.1 \% ; P=.03)$. There was no significant variation in use or timing of DAAs in any clinical vignettes based on institution type (transplant center vs other), region, or provider experience (number of patients treated with HCV or HCC).

\section{Discussion}

To the best of our knowledge, this study represents the first nationally representative survey assessing hepatology provider attitudes and practice patterns regarding DAA use in patients with HCC. Although there was variation in provider beliefs regarding HCC recurrence risk after DAA therapy, we found that most still believed DAAs are beneficial and likely reduce mortality in patients with complete response to HCC treatment. Accordingly, nearly all providers reported recommending DAA therapy in patients with early HCC undergoing curative treatment; however, fewer providers recommended DAA therapy in those with intermediate- or advanced-stage HCC undergoing palliative therapies.

In contrast to the strong data showing reduced risk of incident de novo HCC after DAA therapy, ${ }^{7-9}$ there are conflicting data about HCC recurrence risk after DAA therapy in patients with a history of HCC. Although data from a multicenter cohort in North America suggested no difference in recurrence risk between DAA-treated and untreated patients, ${ }^{11}$ most studies share similar notable limitations: retrospective study design, heterogeneity of tumor burden and treatment leading to complete response, potential for misclassification of complete response, and ascertainment bias for recurrence given lack of a surveillance protocol. Therefore, there is a lack of consensus among professional society guidance statements regarding timing of DAA therapy in patients with a history of HCC. The American Association for the Study of Liver Diseases practice guidance recommends DAA therapy after 3-6 months if no evidence of recurrence, ${ }^{16}$ whereas an American Gastroenterological Association clinical practice update highlights a lack of sufficient data to determine if there is increased or decreased recurrence risk, ${ }^{17}$ and the European Association for the Study of the Liver guidelines state that it remains unclear if DAA therapy increases recurrence but advises caution in this population. ${ }^{18}$ This is reflected in the variation in perceived risk of HCC recurrence after DAA therapy among providers in our survey. However, 

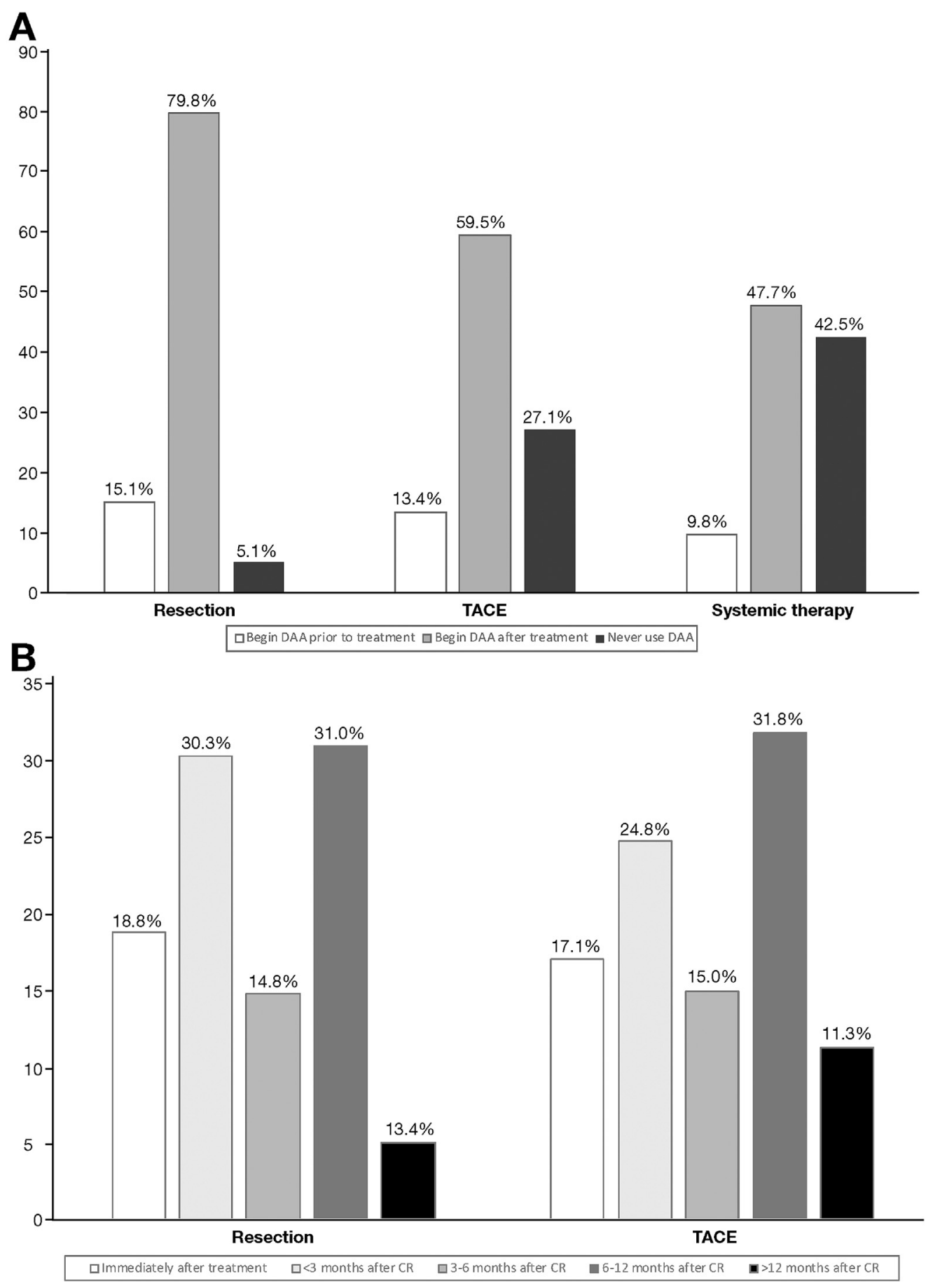

Figure 2. $(A)$ Provider practice patterns for DAA treatment in patients with $\mathrm{HCC}$, according to HCC treatment. (B) Provider practice patterns for timing of DAA treatment in patients with $\mathrm{HCC}$, according to $\mathrm{HCC}$ treatment. $\mathrm{CR}$, complete response; TACE, transarterial chemoembolization.

most providers still believed that DAA therapy would be of overall benefit in patients with HCC. This may be caused, in part, by beliefs that DAAs have a favorable side effect profile or by strong patient preference for viral eradication. Independent of recurrence risk, DAA therapy may improve liver dysfunction and thereby reduce mortality. In fact, hepatic decompensation, not HCC recurrence, is the major driver of mortality in patients with a history of HCC who achieved complete response. ${ }^{19}$ Recent data suggest DAAs may reduce hepatic decompensation and improve overall survival among patients with early stage HCC who achieved complete response from resection or ablation. ${ }^{20}$ These evolving data suggest that patients with confirmed HCC complete response likely benefit from DAA therapy, although further data with larger sample sizes and more heterogeneous tumor burden are needed to confirm the potential benefit of DAA therapy against the competing risk of liver-related mortality on overall survival.

Provider recommendations for DAA therapy differed by HCC stage and treatment type, with providers being less likely to recommend DAAs in advanced patients with HCC undergoing palliative treatments than in early stage patients undergoing curative therapy. This variation is 
Table 4. Timing of DAA Therapy Initiation in Patients With HCC, Stratified by HCC Treatment $(n=279)$

\begin{tabular}{lc}
\hline \multicolumn{1}{c}{ Provider practice } & $\mathrm{N}(\%)$ \\
\hline Timing of DAA initiation in patient with BCLC stage A & \\
HCC undergoing surgical resection & \\
Shortly after resection (before imaging showing CR) & $52(18.8)$ \\
$<3$ mo after CR & $84(30.3)$ \\
$3-6$ mo after CR & $41(14.8)$ \\
6-12 mo after CR & $86(31.1)$ \\
$>12$ mo after CR & $14(5.0)$ \\
Timing of DAA in patient with BCLC stage B HCC & \\
undergoing TACE & \\
Shortly after TACE (before imaging showing CR) & $47(17.2)$ \\
<3 mo after CR & $68(24.8)$ \\
3-6 mo after CR & $41(15.0)$ \\
6-12 mo after CR & $87(31.7)$ \\
$>12$ mo after CR & $31(11.3)$ \\
Timing of DAA in patient with BCLC stage C HCC & \\
undergoing systemic therapy & \\
Begin DAA at time of initiating systemic therapy & $27(9.8)$ \\
Start DAA if partial response & $34(12.4)$ \\
Start DAA if complete response & $78(28.4)$ \\
Would start DAA if develops liver dysfunction, even if & $19(6.9)$ \\
active HCC & $117(42.5)$ \\
Would never start DAA & \\
Timing of DAA therapy in liver transplant candidate with & \\
HCC & \\
Routinely treat pretransplant on waitlist & $115(42.5)$ \\
Case-by-case basis & $26(9.5)$ \\
Routinely treat with DAA within 3 mo post-transplant & $118(43.0)$ \\
Routinely defer DAA >6 mo post-transplant &
\end{tabular}

BCLC, Barcelona Clinic Liver Cancer; CR, complete response; DAA, directacting antiviral; HCC, hepatocellular carcinoma; TACE, transarterial chemoembolization.

presumably driven by the perceived likelihood of achieving a complete response to HCC therapy and the competing risk of HCC-related mortality in patients with more advanced HCC. However, it is possible that DAA therapy in patients with intermediate- or advanced-stage HCC may still be beneficial as expected survival for these patients continues to improve. For example, the median survival for Child Pugh A patients with intermediate stage HCC is approaching 3 years, ${ }^{21,22}$ whereas median survival for advanced-stage patients with HCC is also improving with new systemic options in first- and second-line settings. ${ }^{23,24}$ Prior small studies have suggested patients with HCC who have achieved SVR have improved prognosis compared with those with active viremia. ${ }^{25}$ The reported variation in practice observed may also have been driven by prior reports of lower SVR rates in patients with active HCC. ${ }^{12,13}$ In fact, nearly twothirds of providers raised this concern in patients with active HCC. The American Association for the Study of Liver Diseases practice guidance for HCV therapy recommends consideration of DAA therapy in patients with expected survival exceeding 1 year, raising the question if this should be considered in patients with intermediate and advanced HCC. ${ }^{26}$ As we aim for HCV elimination in all populations, this remains 1 of the few subgroups in whom the benefit of DAA treatment remains unclear.
We observed variation in timing of DAA initiation for all HCC treatment modalities, with some providers treating HCV shortly after HCC complete response and others deferring for several months. We did not find any factor associated with timing of DAA therapy, including a lack of association with provider type, years in practice, or geographic region; this variation may reflect provider knowledge and attitudes that were not included in our survey. There are conflicting data about the importance of DAA timing when considering HCC recurrence risk. Some studies have reported increased recurrence risk with early DAA treatment after HCC complete response when compared with deferred DAA treatment; however, this finding has not been replicated in subsequent studies. ${ }^{10,11}$ An American Gastroenterological Association clinical practice update recommends deferring DAA therapy for 4-6 months following HCC treatment based on the importance of confirming complete response rather than a concern about increasing HCC recurrence risk. $^{17}$ Given the imperfect sensitivity of imaging to detect small HCC lesions and the nonurgent nature of HCV therapy, this seems like a reasonable approach for timing of DAA therapy after HCC complete response.

The important consideration of DAA timing in the context of liver transplantation may have influenced provider responses to the survey. More than half of the providers reported their practice is to routinely delay HCV treatment until the post-transplant period in patients with HCC (who may be undergoing locoregional therapy). This strategy allows for expansion of the donor pool by increasing use of organs from HCV-positive donors, in light of data showing excellent graft and overall survival. ${ }^{27}$ This may have produced variation in practice patterns for the patient undergoing locoregional therapy in the second clinical vignette. Although our intent was to present a patient beyond Milan Criteria, some respondents may have deferred HCV therapy with downstaging and transplantation in mind.

Lastly, our study addressed patterns of HCC surveillance after DAA treatment and subsequent SVR. Although risk of incident HCC is reduced following HCV cure, it is not zero and a proportion of patients will still develop HCC. ${ }^{28}$ Thus, continued surveillance is recommended in all patients with HCV cirrhosis even after SVR. Almost all providers in our study reported continuing surveillance with imaging $\pm \alpha$-fetoprotein every 6 months in patients with cirrhosis. Interestingly, $95 \%$ also reported performing surveillance in at least some patients with F3 fibrosis after SVR, with more than $60 \%$ performing surveillance in all F3 patients. Kanwal et al $^{9}$ demonstrated the risk of HCC is very low $(\sim 0.1 \%)$ in patients without cirrhosis except for those with Fib-4 $>3.25$ (indicating advanced fibrosis), where the annual risk approaches $1 \%$, calling into question the benefit and costeffectiveness of surveillance in patients with F3 fibrosis after SVR. ${ }^{29}$ Notably, some patients labeled as having F3 fibrosis may be misclassified and truly have cirrhosis, suggesting surveillance may be needed in selected 
patients. Predictive models may be useful to identify patients with F3 fibrosis at highest risk of HCC after SVR who could benefit from HCC surveillance. ${ }^{30}$

Strengths of this study include its large sample size and high response rate; however, we acknowledge that our study has limitations. First, the survey was distributed using a convenience sample of tertiary academic centers, with $>98 \%$ having multidisciplinary tumor boards, which may limit generalizability to other practice settings, including community practices, safety-net health systems, and Veterans Affairs hospitals. However, we attempted to mitigate this concern by sampling sites from various US regions and including different provider types (MD/DOs and APPs). Second, the survey was performed and reflects practice patterns in the United States, which may differ from approaches in Europe and Asia. Third, nonhepatology providers involved in HCV treatment, including infectious disease and internal medicine providers, were not included in our sampling frame. Fourth, our results may be limited by response bias, in which providers report how they should practice rather than their actual practice; and nonresponse bias, in which providers who feel more comfortable managing HCV and HCC may be more likely to respond. Fifth, decisions and timing of HCV and HCC treatment are often considered in the context of transplant candidacy, which was not explicitly addressed in some cases and may have influenced provider responses. Similarly, other details that were not explicitly addressed may have impacted providers' interpretation of the clinical vignettes, and thereby explain some observed variation in provider responses. Sixth, we did not address patient preferences (regarding HCV treatment, HCC treatment, and/or transplantation), which play an important role in shared decision-making. Finally, the previously discussed limitations highlight the complexity of decisions about timing of HCV treatment in patients with HCC, thus all potential management options may not have been available for some questions.

In conclusion, in this nationwide survey study of hepatology providers, we found variation in attitudes and practice patterns regarding the use of DAAs in patients with HCC. Our findings highlight a need for highquality data characterizing the risks and benefits of DAA therapy in patients with a history of HCC after complete response and those with active HCC. These data can inform guideline recommendations to help improve and standardize clinical practice for patients with HCV and HCC.

\section{Supplementary Material}

Note: To access the supplementary material accompanying this article, visit the online version of Clinical Gastroenterology and Hepatology at www.cghjournal.org, and at https://doi.org/10.1016/j.cgh.2019.07.042.

\section{References}

1. Mittal S, El-Serag HB. Epidemiology of hepatocellular carcinoma: consider the population. J Clin Gastroenterol 2013; 47(Suppl):S2-S6.

2. Ryerson AB, Eheman CR, Altekruse SF, et al. Annual report to the nation on the status of cancer, 1975-2012, featuring the increasing incidence of liver cancer. Cancer 2016;122:1312-1337.

3. Goldberg D, Ditah IC, Saeian K, et al. Changes in the prevalence of hepatitis C virus infection, nonalcoholic steatohepatitis, and alcoholic liver disease among patients with cirrhosis or liver failure on the waitlist for liver transplantation. Gastroenterology 2017;152:1090-1099.

4. Chhatwal J, Kanwal F, Roberts MS, et al. Cost-effectiveness and budget impact of hepatitis $\mathrm{C}$ virus treatment with sofosbuvir and ledipasvir in the United States. Ann Intern Med 2015; 162:397-406.

5. Foster GR, Irving WL, Cheung MC, et al. Impact of direct acting antiviral therapy in patients with chronic hepatitis C and decompensated cirrhosis. J Hepatol 2016;64:1224-1231.

6. van der Meer AJ, Veldt BJ, Feld JJ, et al. Association between sustained virological response and all-cause mortality among patients with chronic hepatitis $\mathrm{C}$ and advanced hepatic fibrosis. JAMA 2012;308:2584-2593.

7. Backus LI, Belperio PS, Shahoumian TA, et al. Impact of sustained virologic response with direct-acting antiviral treatment on mortality in patients with advanced liver disease. Hepatology 2019;69:487-497.

8. Ioannou GN, Green PK, Berry K. HCV eradication induced by direct-acting antiviral agents reduces the risk of hepatocellular carcinoma. J Hepatol 2018;68:25-32.

9. Kanwal F, Kramer J, Asch SM, et al. Risk of hepatocellular cancer in HCV patients treated with direct-acting antiviral agents. Gastroenterology 2017;153:996-1005.

10. Reig M, Mariño Z, Perelló C, et al. Unexpected high rate of early tumor recurrence in patients with HCV-related HCC undergoing interferon-free therapy. J Hepatol 2016;65:719-726.

11. Singal AG, Rich NE, Mehta N, et al. Direct-acting antiviral therapy not associated with recurrence of hepatocellular carcinoma in a multicenter North American cohort study. Gastroenterology 2019;156:1683-1692.

12. Prenner SB, VanWagner LB, Flamm SL, et al. Hepatocellular carcinoma decreases the chance of successful hepatitis $\mathrm{C}$ virus therapy with direct-acting antivirals. J Hepatol 2017;66:1173-1181.

13. Beste LA, Green PK, Berry K, et al. Effectiveness of hepatitis $C$ antiviral treatment in a USA cohort of veteran patients with hepatocellular carcinoma. J Hepatol 2017;67:32-39.

14. Dalton-Fitzgerald E, Tiro J, Kandunoori P, et al. Practice patterns and attitudes of primary care providers and barriers to surveillance of hepatocellular carcinoma in patients with cirrhosis. Clin Gastroenterol Hepatol 2015;13:791-798.

15. Simmons OL, Feng $\mathrm{Y}$, Parikh ND, et al. Primary care provider practice patterns and barriers to hepatocellular carcinoma surveillance. Clin Gastroenterol Hepatol 2019;17:766-773.

16. Marrero JA, Kulik LM, Sirlin CB, et al. Diagnosis, staging, and management of hepatocellular carcinoma: 2018 practice guidance by the American Association for the Study of Liver Diseases. Hepatology 2018;68:723-750.

17. Singal AG, Lim JK, Kanwal F. AGA Clinical practice update on interaction between oral direct-acting antivirals for chronic hepatitis C infection and hepatocellular carcinoma: expert review. Gastroenterology 2019;156:2149-2157. 
18. EASL Clinical Practice Guidelines. Management of hepatocelIular carcinoma. J Hepatol 2018;69:182-236.

19. Cabibbo G, Petta S, Barbara M, et al. Hepatic decompensation is the major driver of death in HCV-infected cirrhotic patients with successfully treated early hepatocellular carcinoma. J Hepatol 2017;67:65-71.

20. Cabibbo G, Celsa C, Calvaruso V, et al. Direct acting antivirals after successful treatment of early hepatocellular carcinoma improve survival in HCV-cirrhotic patients. J Hepatol 2019; 71:265-273.

21. Lencioni R, de Baere $T$, Soulen MC, et al. Lipiodol transarterial chemoembolization for hepatocellular carcinoma: a systematic review of efficacy and safety data. Hepatology 2016; 64:106-116.

22. Kulik L, El-Serag HB. Epidemiology and management of hepatocellular carcinoma. Gastroenterology 2019;156:477-491.

23. Kudo M, Finn RS, Qin S, et al. Lenvatinib versus sorafenib in first-line treatment of patients with unresectable hepatocellular carcinoma: a randomised phase 3 non-inferiority trial. Lancet 2018;391:1163-1173.

24. Abou-Alfa GK, Meyer T, Cheng A-L, et al. Cabozantinib in patients with advanced and progressing hepatocellular carcinoma. N Engl J Med 2018;379:54-63.

25. Bruno S, Di Marco V, lavarone M, et al. Improved survival of patients with hepatocellular carcinoma and compensated hepatitis $\mathrm{C}$ virus-related cirrhosis who attained sustained virological response. Liver Int 2017;37:1526-1534.

26. Panel AlHG, Chung RT, Davis GL, et al. Hepatitis C guidance: AASLD-IDSA recommendations for testing, managing, and treating adults infected with hepatitis C virus. Hepatology 2015; 62:932-954.

27. Cotter TG, Paul S, Sandikci B, et al. Increasing utilization and excellent initial outcomes following liver transplant of HCVviremic donors into HCV-negative recipients. Hepatology 2019;69:2381-2395.

28. Kanwal F, Kramer JR, Asch SM, et al. Long-term risk of hepatocellular carcinoma in HCV patients treated with direct acting antiviral agents. Hepatology 2020;71:44-55.

29. Zangneh HF, Wong WW, Sander B, et al. Cost-effectiveness of hepatocellular carcinoma surveillance after a sustained virologic response to therapy in patients with $\mathrm{HCV}$ infection and advanced fibrosis. Clin Gastroenterol Hepatol 2019; $17: 1840-1849$.
30. Ioannou GN, Green PK, Beste LA, et al. Development of models estimating the risk of hepatocellular carcinoma after antiviral treatment for hepatitis C. J Hepatol 2018;69:1088-1098.

\section{Reprint requests}

Address requests for reprints to: Nicole E. Rich, MD, Division of Digestive and Liver Diseases, University of Texas Southwestern, 5959 Harry Hines Boulevard, POB 1, Suite 420, Dallas, Texas 75390-8887. e-mail: nicole.rich@ utsouthwestern.edu; fax: (214) 645-6294.

\section{Conflicts of interest}

These authors disclose the following: Ponni Perumalswami has received grant support for research from Gilead. Naim Alkhouri is on speaker's bureau for Gilead, Exelixisi, Eisai, AbbVie, Salix, Intercept, Dova, Shionogi, and Alexion; served on advisory boards for Pfizer, Gilead, Intercept, Eisai, Exelixis, Dova, Shionogi, and Centurion; and received research funding from Gilead, Allergan, Intercept, Genfit, Cirius, Madrigal, Enyo, Inventiva, Hanmi, Novartis, BMS, and Enanta. Neehar Parikh serves as a consultant to Exelixis and Bristol-Myers Squibb; has served on advisory boards for Eisai, Exelixis, Wako, and Bayer and received research funding from Bayer and Target Pharmasolutions. Neil Mehta has received research funding from Wako Diagnostics. Reena Salgia is on speaker's bureau for Bayer; and has served on advisory boards for Bayer Eisai, and Exelixis. Laura Kulik is on speaker's bureau for Eisai, Gilead, and Dova; and serves as an advisory board member for BMS, Eisai, Bayer, and Exelixis. James Hanje is on speaker's bureau for Salix and Intercept; and served on advisory boards for Gilead. Anjana Pillai serves as a consultant and is on speaker's bureau for Eisai and BTG. Robert Wong is on the speaker's bureau, served as consultant and on advisory boards, and has received research funding from Gilead; has received research funding from Abbvie; and was on the speaker's bureau for Bayer. Shaun Chandna has served on an advisory board for Dova Pharmaceuticals; has received sponsored travel for research support from Genfit and Covance; and is on the speaker's bureau for Focus Medical Communications, LLC. Catherine Frenette is on speaker's bureaus for Bayer, Bristol Meyers Squibb, Gilead, Merck, Abbvie, and Eisai, served on advisory boards for Gilead, Eisai, and Wako; served as a consultant for Bayer and Gilead; and received research funding from Bayer. Sanjaya Satapathy has received research support from Gilead and Bayer; and has served on advisory boards or as a consultant for Abbvie and Gilead. Parvez Mantry is on speaker's bureaus and served on advisory boards for Gilead Abbvie, Bayer, BMS, Eisai, Merck, and BTG; and has received research funding from Gilead and Sirtex. Binu John receives research support from Eisai, Bristo Meyers Squibb, Bayer, Exact Sciences, and Varian; and has served on advisory boards for Gilead and Eisai. Michael Leise has received research funding from Abbvie. Nayan Patel has served on advisory boards for Gilead. Z. Gordon Jiang has served as a consultant to Boehringer Ingelheim. Amit Singal was on speaker's bureau for Gilead, Bayer, and Bristol Meyers Squibb; has served on advisory boards for Gilead, Abbvie, Bayer, Eisai, Bristol Meyers Squibb, Wako Diagnostics, and Exact Sciences; serves as a consultant to Bayer, Eisai, Exelixis, Roche, Exact Sciences, and Glycotest; and has received research funding from Gilead and Abbvie. The remaining authors disclose no conflicts.

\section{Funding}

Andrew M. Moon's research is in part supported by National Institutes of Health T32 DK007634. Amit G. Singal's research is in part supported by National Institutes of Health R01CA222900. The content is solely the responsibility of the authors and does not necessarily represent the official views of the National Institutes of Health. 